\title{
Perturbative structure of two- and four-point functions of color charge in a non-Gaussian small- $x$ action
}

\author{
Andre V. Giannini ${ }^{1,2}$, Yasushi Nara ${ }^{3, a}$ \\ ${ }^{1}$ Instituto de Física Gleb Wataghin, Universidade Estadual de Campinas, R. Sérgio Buarque de Holanda, 777, Campinas 13083-859, Brazil \\ ${ }^{2}$ Instituto de Física, Universidade de São Paulo, Rua do Matão 1371, São Paulo, SP 05508-090, Brazil \\ ${ }^{3}$ Akita International University, Yuwa, Akita 010-1292, Japan
}

Received: 15 November 2021 / Accepted: 10 January 2022 / Published online: 4 February 2022

(C) The Author(s) 2022

\begin{abstract}
We compute the perturbative expansion of the two- and four-point functions of color charges in the Color Glass Condensate framework considering the quartic correction to the McLerran-Venugopalan (MV) model of Gaussian color charge fluctuations. Expressions for these correlators in the perturbative expansion for small and large non-Gaussian color charge fluctuations are derived for arbitrary orders in perturbation theory. We explicitly show that the perturbative series does not converge at higher orders as expected. We apply the Borel-Padé resummation method to our problem to construct a convergent series. It is shown that the fully non-perturbative solution can be described by the BorelPadé approximants constructed from the first few terms of the perturbative series for small non-Gaussian fluctuations.
\end{abstract}

\section{Introduction}

The color glass condensate (CGC) effective theory [1,2] has been successively used in understanding initial particle production processes at high energy hadronic collisions and the initial phase of collisions of heavy nuclei. The MclerranVenugopalan (MV) model [3-5] of color charge fluctuations in nuclei assumes a Gaussian weight function, which is believed to be a good approximation for large systems. One expects that non-Gaussian corrections to the MV model may become important for describing color charge fluctuations in small collision systems. These corrections may also be relevant to the dijet correlations. In particular, the recent observation of a "ridge" in two-particle correlations from high-multiplicity $p p$ and $p A$ collisions [6-17] motivates us to consider the non-Gaussian effects. The ridge for $\mathrm{Au}+\mathrm{Au}$ collisions [18-22] is believed to be due to collective effects and the signature of the formation of quark-gluon plasma,

\footnotetext{
a e-mail: nara@aiu.ac.jp (corresponding author)
}

which can be described by hydrodynamic evolution [23,24]. An outstanding question remains: whether the ridge effect observed in collisions involving small systems is due to collective effects or initial state dynamics [23-25].

Non-Gaussian corrections for the weight function have been derived up to the fourth-order in the color charges [2628]. In Ref. [28], the two- and four-point functions of the color charges are computed at leading order in the regime where the quartic term is assumed to be a small perturbation, and showed that infrared behavior of the leading connected two-particle production diagram is different from the case of a quadratic action. We discussed the properties of the nonperturbative solution in Ref. [29] for arbitrarily large nonGaussian fluctuations. The non-Gaussian action could be employed for generating initial conditions for the JIMWLK evolution equation [30-39]; in particular, it would provide corrections to the JIMWLK evolution equation for the fourpoint function of color charges not considered in [40].

In this paper, we extend the work of Ref. [28] by computing these color charge correlators at higher orders in perturbation theory. We explicitly compute diagrams up to nextto-next-leading-order (NNLO). Computing each diagram is helpful to understand the structure of the theory. However, when going to several orders beyond the leading one, it may not be useful anymore to see the complicated graphs. Instead, we propose a general method to compute two- and four-point functions at any order in the perturbation. It is well known that the perturbative expansion in quantum mechanics and quantum field theory often has zero radius of convergence [4144]. The asymptotic character of perturbation theory is also suggested by the fact that the number of Feynman diagrams at order $n$ typically grows factorially. In general, perturbative solution yields extremely good accuracy, e.g. quantum electrodynamics, but it will diverge at the terms of order of the inverse of coupling constant. We shall show that our perturbative series is also divergent, and the optimal order for 
truncating the perturbative series depends on the values of coupling constant.

The Borel algorithm in perturbation theory is a resummation method to construct a convergent perturbative series, see e.g. $[45,46]$. Based on the Borel method, several resummation methods are proposed $[47,48]$. We will apply a widely used one for our problem: the Borel-Padé resummation method [47]. In this method, a Padé approximant replaces the Borel-transformed series expansion by a rational function whose numerator and denominator are chosen so that its power series expansion agrees with the original power series up to the term whose degree is equal to the sum of the degrees of the numerator and the denominator of the rational function. We shall show that almost exact solutions can be obtained by taking only the first few terms of the divergent series.

This paper is organized as follows. After describing the setup of our problem in Sect. 2, we work out perturbative expressions for the limit of small non-Gaussian fluctuations and compare its results to the full non-perturbative calculation in Sect. 3. A formulation of the perturbative series in the limit of large non-Gaussian fluctuations is given in Sect. 4. In Sect. 5, we show the results of the Borel-Padé approximant for our problem. The conclusion is given in Sect. 6.

\section{Color charge averaging for small/large deviations from the MV model}

We consider the asymptotic expansion of the quartic correction to the MV model $^{1}$ [28]

$$
\begin{aligned}
& \langle\mathcal{O}[\rho]\rangle \equiv \frac{\int \mathcal{D} \rho \mathcal{O}[\rho] e^{-S_{G}-\int d^{2} w \rho_{w}^{4} / \kappa}}{\int \mathcal{D} \rho e^{-S_{G}-\int d^{2} w \rho_{w}^{4} / \kappa}} \\
& =\frac{\int \mathcal{D} \rho \mathcal{O}[\rho] e^{-S_{G}} \sum_{k=0}^{\infty} \frac{1}{k !}\left(-\frac{1}{\kappa} \int d^{2} w \rho_{w}^{4}\right)^{k}}{\int \mathcal{D} \rho e^{-S_{G}} \sum_{k=0}^{\infty} \frac{1}{k !}\left(-\frac{1}{\kappa} \int d^{2} w \rho_{w}^{4}\right)^{k}}
\end{aligned}
$$

where $S_{G}=\int d^{2} x \rho_{x}^{2} / 2 \mu^{2}$ denotes the Gaussian action. On the other hand, in the regime of large non-Gaussian fluctuations, one may consider the expansion of the action as:

$$
\begin{aligned}
& \int \mathcal{D} \rho_{x} \exp \left[-\frac{Z \rho_{x}^{2}}{2 \bar{\mu}^{2}}-\frac{\rho_{x}^{4}}{\kappa}\right] \\
& =\int \mathcal{D} \rho_{x} \sum_{k=0}^{\infty} \frac{1}{k !}\left(-\frac{Z \rho_{x}^{2}}{2 \bar{\mu}^{2}}\right)^{k} \exp \left[-\frac{\rho_{x}^{4}}{\kappa}\right],
\end{aligned}
$$

\footnotetext{
1 We note that we absorbed a factor 3 into the definition of $\kappa: \kappa=\kappa_{4} / 3$, as compared to [29]. We also inverted the notation for the coupling in the MV model and the quadratic term in the non-Gaussian action to make comparisons with [28] easier: $\mu$ in this paper is the coupling of the quadratic term in the non-Gaussian action while $\bar{\mu}$ is the renormalized color charge appearing in the MV model.
}

where $Z=\bar{\mu}^{2} / \mu^{2}$ is the renormalization factor, and $\bar{\mu}$ is the coefficient of the two-point function in the MV model, i.e., the renormalized color charge:

$\left\langle\rho^{a}(x) \rho^{b}(y)\right\rangle=\left\langle\rho_{x}^{a} \rho_{y}^{b}\right\rangle=\delta^{a b} \delta(x-y) \bar{\mu}^{2}$.

Following [28,29], we compute the functional integral in lattice regularization, assuming a square lattice with $N_{s} \times N_{s}$ sites of length $a$. In the case of a local operator, which is the one we consider here, the color charge average in the $\mathrm{SU}\left(N_{c}\right)$ theory can be written as:

$\left\langle\mathcal{O}_{r}\right\rangle=\frac{\int d r r^{N_{c}^{2}-2} \mathcal{O}_{r} e^{-W_{r}}}{\int d r r^{N_{c}^{2}-2} e^{-W_{r}}}$,

where $r^{2}=\sum_{a=1}^{N_{c}^{2}-1} \rho_{x}^{a} \rho_{x}^{a}$ and

$W_{r}=\frac{a^{2} r^{2}}{2 \mu^{2}}+\frac{a^{2} r^{4}}{\kappa}$.

In the next section, we compute the two- and four-point function of color charges in the limits where (i) Eq. (5) is dominated by its quadratic term, and (ii) Eq. (5) is dominated by its quartic term, corresponding to the regimes of small and large non-Gaussian fluctuations, respectively, and compare the result from different orders in perturbation to the nonperturbative result.

\section{Perturbation theory in the limit of small non-Gaussian fluctuations}

A leading order (LO) calculation in $1 / \kappa$ for two- and fourpoint function of color charges has already been presented in [28]. We start by summarizing their result, then extend calculations to next-to-leading order (NLO) and next-tonext-to-leading order (NNLO). Then, we present the structure of these correlators at $\mathrm{N}^{n} \mathrm{LO}$ order in perturbation theory.

\subsection{Leading order}

We summarize the LO result for the two- and four-point function [28]. The color factors of the basic diagrams contributing at this order are shown in Fig. 1, where $x, y, u$, and $v$ represent coordinates in position space and $a, b, c$, and $d$ represent color indexes. We suppress color indexes in other figures to avoid cluttered diagrams.

From Eq. (1), the two-point function at the order $1 / \kappa$

$$
\left\langle\rho_{x}^{a} \rho_{y}^{b}\right\rangle=\frac{\int \mathcal{D} \rho \rho_{x}^{a} \rho_{y}^{b} e^{-S_{G}}\left[1-\frac{1}{\kappa} \int d^{2} w \rho_{w}^{4}\right]}{\int \mathcal{D} \rho e^{-S_{G}}\left[1-\frac{1}{\kappa} \int d^{2} w \rho_{w}^{4}\right]},
$$




$$
\left.\right|_{(x, a)} ^{(y, b)}=\frac{\mu^{2}}{a^{2}} \delta^{a b} \delta_{x y} \bigodot_{w}=\left.\left(N_{c}^{4}-1\right) N_{s} \frac{\mu^{4}}{a^{2}}\right|_{(x, a)} ^{(y, b)}=4\left(N_{c}^{2}+1\right) \frac{\mu^{6}}{a^{4}} \delta^{a b} \delta_{x y}
$$
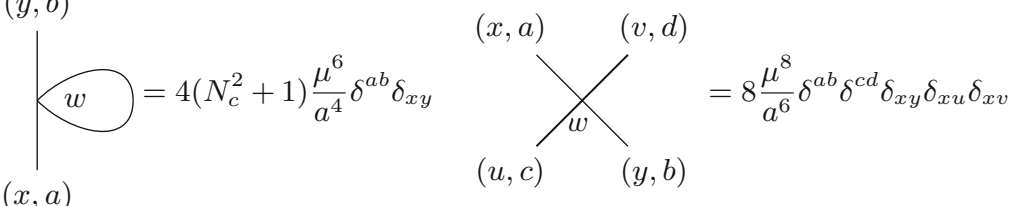

Fig. 1 The color factor for diagrams contributing at LO for the two- and four-point function of color charges

can be expressed diagrammatically as:

$$
\begin{aligned}
& \left\langle\rho_{x}^{a} \rho_{y}^{b}\right\rangle=\left(\left.\right|_{x} ^{y}-\frac{1}{\kappa}\left[\left.\right|_{x} ^{y} \delta w+\langle w]\right)\right. \\
& \times\left(1-\frac{1}{\kappa} \bigcirc w\right)^{-1}
\end{aligned}
$$

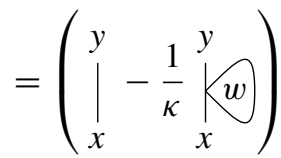

$$
\begin{aligned}
& =\mu^{2} \frac{\delta^{a b} \delta_{x y}}{a^{2}}\left(1-4\left(N_{c}^{2}+1\right) \frac{\mu^{4}}{\kappa a^{2}}\right) \text {. }
\end{aligned}
$$

Given that the two-point function of color charges must be the same in both the MV model and its non-Gaussian extension, we identify the renormalized the average charge squared at order $\mathcal{O}(1 / \kappa)$ as:

$\bar{\mu}^{2}=\mu^{2}\left[1-4 \frac{\mu^{4}}{\kappa a^{2}}\left(N_{c}^{2}+1\right)\right]$.

Thus, the two-point function reads

$\left\langle\rho_{x}^{a} \rho_{y}^{b}\right\rangle=\frac{\delta^{a b} \delta_{x y}}{a^{2}} \bar{\mu}^{2}$.

The four-point function at leading order in $1 / \kappa$ evaluates to:

$$
\begin{aligned}
& \left\langle\rho_{x}^{a} \rho_{y}^{b} \rho_{u}^{c} \rho_{v}^{d}\right\rangle \\
& =\left(\left.\left.\right|_{x} ^{y}\right|_{u} ^{v}-\frac{1}{\kappa}[\left.\left.\right|_{x} ^{y}\right|_{u} ^{v} 8 w+\left.\right|_{x} ^{y} w \bigvee_{u}^{v} \sum_{y}^{x} \underbrace{v}]\right) \\
& \times\left(1-\frac{1}{\kappa} \bigcirc w\right)^{-1} \\
& =\left(\left.\left.\right|_{x} ^{y}\right|_{u} ^{v}-\frac{1}{\kappa}[\left.\right|_{x} ^{y} w \aleph_{u}^{v}+\underbrace{x}_{y}]\right) \\
& =\frac{\mu^{4}}{a^{4}}\left(\delta^{a b} \delta_{x y} \delta^{c d} \delta_{u v}+\delta^{a c} \delta_{x u} \delta^{b d} \delta_{y v}+\delta^{a d} \delta_{x v} \delta^{b c} \delta_{y u}\right) \\
& \times\left[1-8 \frac{\mu^{4}}{\kappa a^{2}}\left(N_{c}^{2}+1\right)\right]
\end{aligned}
$$

$$
-8 \frac{\mu^{8}}{\kappa a^{6}}\left(\delta^{a b} \delta^{c d}+\delta^{a c} \delta^{b d}+\delta^{a d} \delta^{b c}\right) \delta_{x y} \delta_{x u} \delta_{u v} .
$$

Equation (10) implies

$\bar{\mu}^{4}=\mu^{4}\left[1-8 \frac{\mu^{4}}{\kappa a^{2}}\left(N_{c}^{2}+1\right)\right]$,

at leading order in $1 / \kappa$. The term $\mu^{8} / \kappa$ is replaced by $\mu^{8} \rightarrow$ $\bar{\mu}^{8}$ as any higher-order contribution in $1 / \kappa$ is discarded at LO. Thus, the four-point function at LO in $1 / \kappa$ becomes

$$
\begin{aligned}
& \left\langle\rho_{x}^{a} \rho_{y}^{b} \rho_{u}^{c} \rho_{v}^{d}\right\rangle=\frac{\bar{\mu}^{4}}{a^{4}}\left(\delta^{a b} \delta_{x y} \delta^{c d} \delta_{u v}\right. \\
& \left.+\delta^{a c} \delta_{x u} \delta^{b d} \delta_{y v}+\delta^{a d} \delta_{x v} \delta^{b c} \delta_{y u}\right)-8 \frac{\bar{\mu}^{8}}{\kappa a^{6}}\left(\delta^{a b} \delta^{c d}\right. \\
& \left.+\delta^{a c} \delta^{b d}+\delta^{a d} \delta^{b c}\right) \delta_{x y} \delta_{x u} \delta_{u v} .
\end{aligned}
$$

\subsection{The next-to-leading order}

We now compute the two- and four-point functions using Eq. (1) at NLO in $1 / \kappa$. The two-point function reads:

$$
\begin{aligned}
\left\langle\rho_{x}^{a} \rho_{y}^{b}\right\rangle & \left(\left.\right|_{x} ^{y}-\left.\frac{1}{\kappa}\right|_{x} ^{y} w+\frac{1}{2 \kappa^{2}}\left[\left.\right|_{x} ^{y}+\left.\right|_{x}\right]\right. \\
& \left.+\prod_{w}^{z}\right]\left.\right|^{2} \frac{\delta^{a b} \delta_{x y}}{a^{2}}\left[1-4 \frac{\mu^{4}}{\kappa a^{2}}\left(N_{c}^{2}+1\right)\right. \\
& +\frac{\mu^{8}}{2 \kappa^{2} a^{4}}\left(32\left(N_{c}^{2}+1\right)^{2}+32\left(N_{c}^{2}+1\right)^{2}\right. \\
& \left.\left.+32 \times 2\left(N_{c}^{2}+1\right)\right)\right] \\
= & \mu^{2} \frac{\delta^{a b} \delta_{x y}}{a^{2}}\left[1-4 \frac{\mu^{4}}{\kappa a^{2}}\left(N_{c}^{2}+1\right)\right. \\
& \left.+32 \frac{\mu^{8}}{\kappa^{2} a^{4}}\left(N_{c}^{2}+1\right)\left(N_{c}^{2}+2\right)\right] .
\end{aligned}
$$


Thus, the color charge squared is renormalized as

$$
\begin{aligned}
\bar{\mu}^{2} \equiv & \mu^{2}\left[1-4 \frac{\mu^{4}}{\kappa a^{2}}\left(N_{c}^{2}+1\right)\right. \\
& \left.+32 \frac{\mu^{8}}{\kappa^{2} a^{4}}\left(N_{c}^{2}+1\right)\left(N_{c}^{2}+2\right)\right] .
\end{aligned}
$$

For the four-point function there are six connected diagrams that contribute at NLO, whose color factors are:
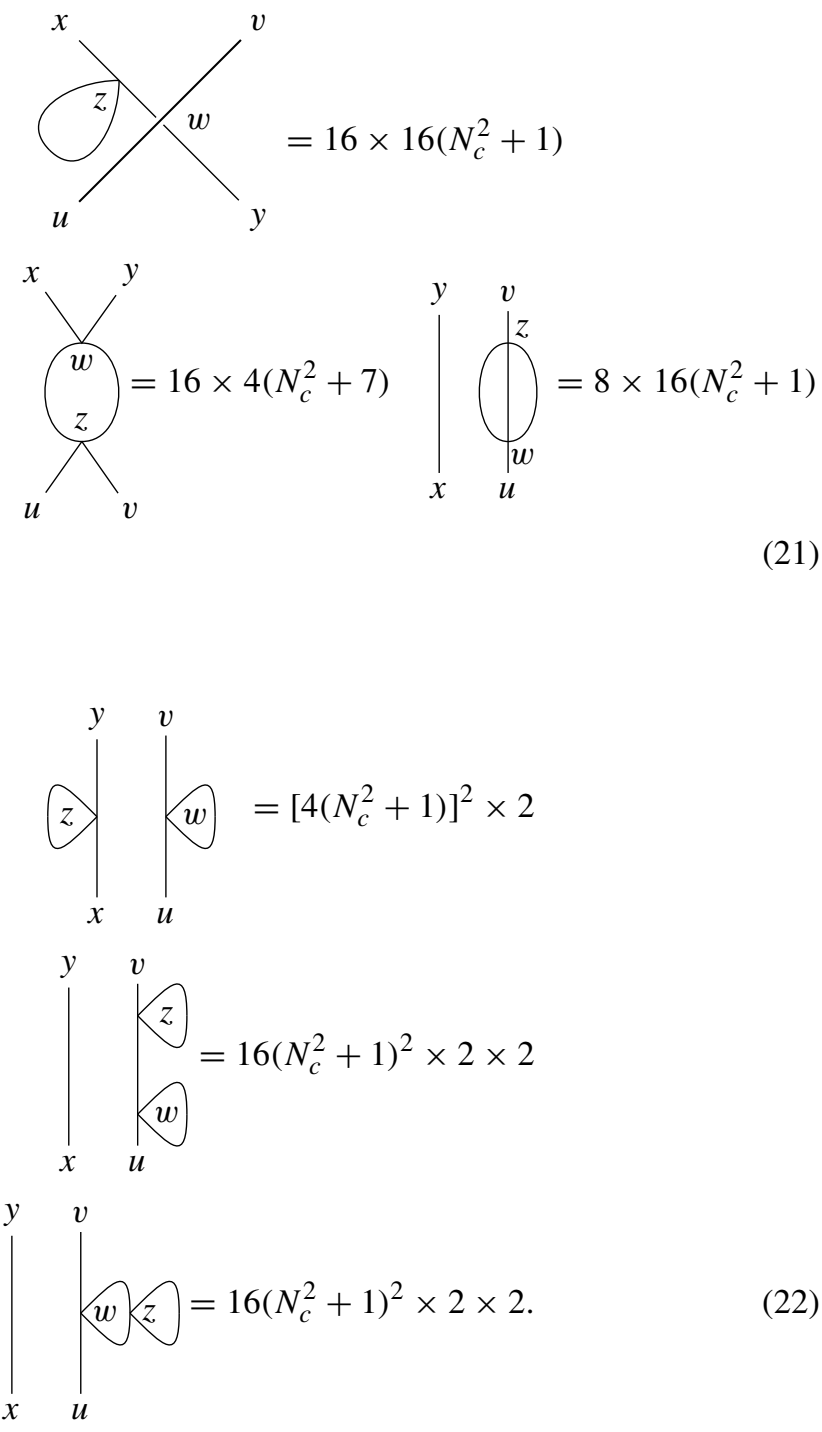

Let us define the four-point function as

$$
\begin{aligned}
& \left\langle\rho_{x}^{a} \rho_{y}^{b} \rho_{u}^{c} \rho_{v}^{d}\right\rangle=\left(\delta^{a b} \delta_{x y} \delta^{c d} \delta_{u v}\right. \\
& \left.\quad+\delta^{a c} \delta_{x u} \delta^{b d} \delta_{y v}+\delta^{a d} \delta_{x v} \delta^{b c} \delta_{y u}\right) A_{\mathrm{dis}} \\
& \quad+\left(\delta^{a b} \delta^{c d}+\delta^{a c} \delta^{b d}+\delta^{a d} \delta^{b c}\right) \delta_{x y} \delta_{x u} \delta_{u v} A_{\mathrm{con}},
\end{aligned}
$$

where the disconnected (MV) part, $A_{\text {dis }}$, must be the square of the two-point function (at the given order in perturbation theory) as seen in the computation at leading order presented in the previous section:

$$
\begin{aligned}
A_{\text {dis }}= & \frac{\mu^{4}}{a^{4}}\left[1-8 \frac{\mu^{4}}{\kappa a^{2}}\left(N_{c}^{2}+1\right)\right. \\
& +64 \frac{\mu^{8}}{\kappa^{2} a^{4}}\left(N_{c}^{2}+1\right)\left(N_{c}^{2}+2\right) \\
& \left.+16 \frac{\mu^{8}}{\kappa^{2} a^{4}}\left(N_{c}^{2}+1\right)^{2}\right]=\frac{\bar{\mu}^{4}}{a^{4}} .
\end{aligned}
$$

The connected part is given by

$$
\begin{aligned}
A_{\text {con }}= & \frac{\mu^{4}}{a^{4}}\left[-8 \frac{\mu^{4}}{\kappa a^{2}}\left(1-\frac{16 \mu^{4}}{\kappa a^{2}}\left(N_{c}^{2}+1\right)\right)\right. \\
& \left.+32 \frac{\mu^{8}}{\kappa^{2} a^{4}}\left(N_{c}^{2}+7\right)\right] .
\end{aligned}
$$

The first term is renormalized by the left-most diagram in Eq. (21) and we can replace $\mu \rightarrow \bar{\mu}$ in the second term as it is already a term of order $\mathcal{O}\left(1 / \kappa^{2}\right)$ :

$A_{\text {con }}=\frac{\bar{\mu}^{4}}{a^{4}}\left[-8 \frac{\bar{\mu}^{4}}{\kappa a^{2}}+32 \frac{\bar{\mu}^{8}}{\kappa^{2} a^{4}}\left(N_{c}^{2}+7\right)\right]$.

An explicit computation of each diagram that contributes for the two- and four-point function of color charge at NNLO $\left(\mathcal{O}\left(1 / \kappa^{3}\right)\right)$ is presented in the Appendix A.

We shall work out the structure of these correlators for an arbitrary order in perturbation theory in the next section. For this purpose, it would be helpful to show Eqs. (27) and (28) in a slightly different way, which can be extend to the evaluation of the renormalized coefficients at $\mathrm{N}^{n} \mathrm{LO}$. The non-vanishing part of the two- and four-point function before and after renormalization may be expressed as

$$
\begin{aligned}
\left\langle\rho_{x}^{a} \rho_{x}^{a}\right\rangle & =\left(N_{c}^{2}-1\right) \frac{\mu^{2}}{a^{2}}\left[1-c x\left(1+a_{1} x\right)\right] \\
& =\left(N_{c}^{2}-1\right) \frac{\mu^{2}}{a^{2}}\left[1-c y\left(1+c_{1} y\right)\right] \\
& =\left(N_{c}^{2}-1\right) \frac{\bar{\mu}^{2}}{a^{2}}, \\
\left\langle\rho_{x}^{a} \rho_{x}^{a} \rho_{x}^{b} \rho_{x}^{b}\right\rangle & =\left(N_{c}^{4}-1\right) \frac{\mu^{4}}{a^{4}}\left(1+a_{1} x+a_{2} x^{2}\right) \\
& =\left(N_{c}^{4}-1\right) \frac{\bar{\mu}^{4}}{a^{4}}\left(1+c_{1} y+c_{2} y^{2}\right),
\end{aligned}
$$

where

$$
\begin{aligned}
c & =4\left(N_{c}^{2}+1\right), \quad a_{1}=-8\left(N_{c}^{2}+2\right), \\
a_{2} & =16\left(5 N_{c}^{4}+24 N_{c}^{2}+31\right), \\
c_{1} & =-8, \quad c_{2}=32\left(N_{c}^{2}+7\right),
\end{aligned}
$$

and $x=\mu^{4} / \kappa a^{2}$ and $y=\bar{\mu}^{4} / \kappa a^{2}$. From Eq. (27), the renormalization condition is obtained as

$\mu^{2}\left[1-c x\left(1+a_{1} x\right)\right]=\bar{\mu}^{2}$ 
from which we have

$x\left[1-c x\left(1+a_{1} x\right)\right]^{2}=y$.

Solving this equation for $x$ up to the next-to-leading order yields:

$x=y\left[1+2 c y+\left(7 c^{2}+2 c a_{1}\right) y^{2}\right]$.

Then, the four-point function can be renormalized as

$$
\begin{aligned}
x\left(1+a_{1} x+a_{2} x^{2}\right)= & y\left[1+\left(a_{1}+2 c\right) y\right. \\
& \left.+\left(7 c^{2}+6 c a_{1}+a_{2}\right) y^{2}\right] .
\end{aligned}
$$

Thus, we also obtained $c_{1}=a_{1}+2 c=-8$ and $c_{2}=7 c^{2}+$ $6 c a_{1}+a_{2}=32\left(N_{c}^{2}+7\right)$.

It is worth recognizing that the two-point function has the same coefficients as the four-point function: $c_{1}$, in the case of NLO. In other words, the coefficients of the four-point function are obtained by dividing the coefficients of the two-point function by $c=4\left(N_{c}^{2}+1\right)$ as expected by the cutting rule. Starting from the vacuum graph at order $1 / \kappa$, we note that the contribution for the two-point function can be obtained by cutting a loop from that graph in all possible ways, as indicated in Eq. (34) (a cut is indicated by the $\times$ symbol). The fully connected graph for the four-point function at order $1 / \kappa$ can be obtained by cutting a loop in the resulting diagram for the two-point function, which has been shown in the case of scalar field theory with $\phi^{4}$ self-interaction [49]:

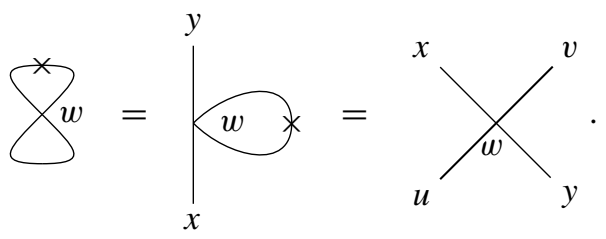

We note that scalar $\phi^{4}$ interaction has the same diagrams as our problem in the perturbative expansion. The corresponding color factor for the second diagram above may be obtained by dividing the vacuum graph by $\left(N_{c}^{2}-1\right)$, then one may multiply it by the $M V$-part of the two-point function: $\mu^{2} \delta^{a b} \delta_{x y} / a^{2}$ to get the $\mathcal{O}(1 / \kappa)$ contribution to the two-point function of color charges. The tadpole diagram always have the color factor $4\left(N_{c}^{2}+1\right)$. This factor disappears after cutting the loop. The $\mathcal{O}(1 / \kappa)$ contribution for the four-point function of color charges can be obtained by dividing the color factor of the $\mathcal{O}(1 / \kappa)$ contribution to the two-point function by $4\left(N_{c}^{2}+1\right)$ and then multiplying it by $\mu^{2} \delta^{a b} \delta_{x y} / a^{2}$.

In Fig. 2, we compare the LO, NLO, and NNLO results with the non-perturbative result for the four point function (left panel) and the solution of the renormalization equation (right panel). We obtain a better agreement as the perturbative order increases. We see that the NNLO result is enough to reproduce the exact solution when the deviation from the Gaussian approximation is less than $10 \%$. In the next section, we will show the $\mathrm{N}^{n} \mathrm{LO}$ results.

\subsection{The $\mathrm{N}^{n} \mathrm{LO}$ order}

We consider a perturbative expansion in $1 / \kappa$ at an arbitrary order. To get a total sum of all diagrams, it is easier to work with the Gaussian integrals rather than by using the Wick contraction. We define $N_{n}$ as

$N_{n}=\frac{\int \prod_{a} d \rho_{x}^{a} \rho_{x}^{n} e^{-S_{G}}}{\int \prod_{a} d \rho_{x}^{a} e^{-S_{G}}}$,

for which we have the recursive expression:

$N_{n+2}=\left(N_{c}^{2}-1+n\right)\left(\frac{\mu}{a}\right)^{2} N_{n}, \quad$ with $N_{0}=1$,

for $n \geq 0$ and even. A derivation of Eq. (36) is presented in the Appendix B. By using $N_{n}$, the two-point and four-point function at $\mathrm{N}^{n} \mathrm{LO}$ can be expressed as:

$$
\begin{aligned}
\left\langle\rho_{x}^{a} \rho_{x}^{a}\right\rangle= & \left(\sum_{k=0}^{n+1} \frac{1}{k !}\left(-\frac{a^{2}}{\kappa}\right)^{k} N_{4 k+2}\right) \\
& \times\left(\sum_{k=0}^{n+1} \frac{1}{k !}\left(-\frac{a^{2}}{\kappa}\right)^{k} N_{4 k}\right)^{-1} \\
= & \left(N_{c}^{2}-1\right) \frac{\mu^{2}}{a^{2}}\left[1-c \sum_{k=0}^{n} a_{k} x^{k+1}\right] \\
\left\langle\rho_{x}^{a} \rho_{x}^{a} \rho_{x}^{b} \rho_{x}^{b}\right\rangle= & \left(\sum_{k=0}^{n+1} \frac{1}{k !}\left(-\frac{a^{2}}{\kappa}\right)^{k} N_{4 k+4}\right)^{-1} \\
& \times\left(\sum_{k=0}^{n+1} \frac{1}{k !}\left(-\frac{a^{2}}{\kappa}\right)^{k} N_{4 k}\right)^{-1} \\
= & \left(N_{c}^{4}-1\right) \frac{\mu^{4}}{a^{4}} \sum_{k=0}^{n+1} a_{k} x^{k},
\end{aligned}
$$

where $x=\mu^{4} / \kappa a^{2}$. Note that the four-point function has the same coefficients $a_{k}$ since it can be obtained by cutting diagrams of two-point function at each order as mentioned in previous sections. The factor $c=4\left(N_{c}^{2}+1\right)$ is a contribution of a loop, which disappears after cutting the loop when calculating the four-point function. The coefficients $a_{k}$ are listed in the Appendix $\mathrm{C}$ up to ten orders in perturbation.

Imposing the condition that the two-point function must be matched with the MV model is written as:

$Z \equiv \frac{\bar{\mu}^{2}}{\mu^{2}}=1-c \sum_{k=0}^{n} a_{k} x^{k+1}$,

where $\bar{\mu}^{2}$ is the renormalized color charge squared appearing in the two-point function in the MV model. The renormalized expression is obtained by substituting

$$
\begin{aligned}
\mu^{2} & =\bar{\mu}^{2}\left(1-c x-c a_{1} x^{2} \cdots\right)^{-1} \\
& =\bar{\mu}^{2}\left(1+c x+\left(c a_{1}+c^{2}\right) x^{2}+\left(c a_{2}+2 c^{2} a_{1}+c^{3}\right) x^{3}\right.
\end{aligned}
$$


Fig. 2 The left panel shows the ratio of the four-point function as a function of $Z$ from LO, NLO, and NNLO calculations for SU(3). The right panel shows the $Z$ dependence of $y=\bar{\mu}^{4} / \kappa a^{2}$. The full squares represent the result of the non-perturbative calculation
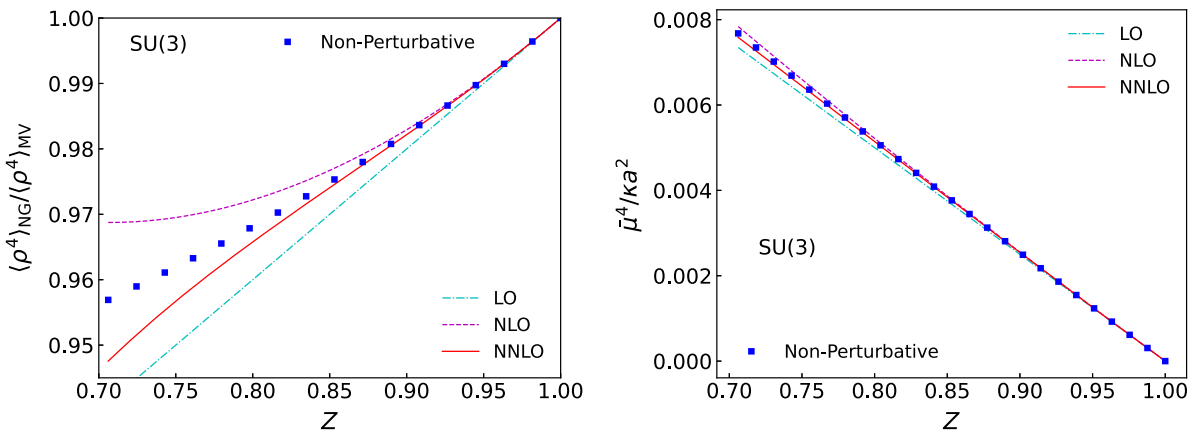

$$
\left.+\left(c a_{3}+2 c^{2} a_{2}+c^{2} a_{1}^{2}+3 c^{3} a_{1}+c^{4}\right) x^{4}+\cdots\right)
$$

successively until all powers of $x$ are replaced by powers of $y=\bar{\mu}^{4} / \kappa a^{2}$ at a desired order so that we obtain the renormalization of Eq. (39):

$Z=1-c \sum_{k=0}^{n} c_{k} y^{k+1}$.

The four-point function can be renormalized following the exact same procedure. After renormalization, Eq. (38) may be written as:

$\left\langle\rho_{x}^{a} \rho_{x}^{a} \rho_{x}^{b} \rho_{x}^{b}\right\rangle=\left(N_{c}^{4}-1\right) \frac{\bar{\mu}^{4}}{a^{4}} \sum_{k=0}^{n+1} c_{k} y^{k}$.

The coefficients $c_{k}$ can be found in the Appendix C. The four-point function of color charges in continuum notation becomes

$$
\begin{aligned}
& \left\langle\rho_{x}^{a} \rho_{y}^{b} \rho_{u}^{c} \rho_{v}^{d}\right\rangle=\bar{\mu}^{4}\left[\delta^{a b} \delta^{c d} \delta(x-y) \delta(u-v)\right. \\
& \times\left(1+\frac{\bar{\mu}^{4}}{\kappa} A_{\operatorname{con}} \delta(x-u)\right) \\
& +\delta^{a c} \delta^{b d} \delta(x-u) \delta(y-v)\left(1+\frac{\bar{\mu}^{4}}{\kappa} A_{\operatorname{con}} \delta(x-y)\right) \\
& \left.+\delta^{a d} \delta^{b c} \delta(x-v) \delta(y-u)\left(1+\frac{\bar{\mu}^{4}}{\kappa} A_{\operatorname{con}} \delta(x-y)\right)\right],
\end{aligned}
$$

where

$A_{\mathrm{con}}=c_{1}+c_{2} y+c_{3} y^{2}+c_{4} y^{3}+\cdots+c_{n+1} y^{n}$

with $y$ determined by solving Eq. (41).

The ratio of the four-point function is given by

$R_{n}=\frac{\left\langle\rho_{x}^{4}\right\rangle}{\left\langle\rho_{x}^{4}\right\rangle_{\mathrm{MV}}}=\sum_{k=0}^{n+1} c_{k} y^{k}=\frac{1-Z}{c y^{\prime}}$,

where $y^{\prime}$ is a solution of Eq. (41) at order $y^{n+2}$. These results suggests that we can sum divergent series:

$R_{\infty}=\sum_{k=0}^{\infty} c_{k} y^{k}=\frac{1-Z}{c y_{\infty}}$ providing that we know a exact solution $y_{\infty}$ for the renormalization equation.

We plot the result from Eq. (45) in Fig. 3 for SU(3) group as a function of $Z$ up to the eighteenth order in the expansion. These ratios go up (down) for $\mathrm{N}^{2 n+1} \mathrm{LO}\left(\mathrm{N}^{2 n} \mathrm{LO}\right)$. Such a behaviour indicates that a large value is added or subtracted at each order. The perturbation in $1 / \kappa$ works up to $Z \sim$ 0.85 , and perturbative solutions strongly disagree with the full solution. The right panel shows the $Z$ dependence of $y=\bar{\mu}^{4} / \kappa a^{2}$. We can see that the perturbative solutions also deviate from the full solution around $Z=0.85$, which is the source of the divergence in the ratio of the four-point functions.

Usually, the partial sum of the asymptotic expansion (order by order) will first approach the true value, then it starts to diverge for larger orders. The perturbative solution is known to be good approximation up to the term of the order of the inverse of coupling constant. Let us estimate what order in the perturbative series the expansion around $1 / \kappa \approx 0$ is optimal (optimal truncation). By using the Stirling approximation, we make an rough estimate for the coefficients:

$$
\begin{aligned}
c_{k} \sim \frac{1}{k !}\left(\frac{a^{2}}{\kappa}\right)^{k} N_{4 k} & =\frac{\Gamma\left(2 k+\left(N_{c}^{2}-1\right) / 2\right)}{\Gamma\left(\left(N_{c}^{2}-1\right) / 2\right)} \frac{(-4 x)^{k}}{k !} \\
& \sim(-16 x)^{k} k !,
\end{aligned}
$$

which grow factorially at large $k$. This gives the order estimate of the optimal number of terms $k_{\text {opt }} \sim 1 /(16 x)=$ $Z^{2} /(16 y)$ [42]. When we substitute the value of $y=0.004$ at $Z=0.84$, the optimal value is $k_{\mathrm{opt}} \approx 11$. For the value of $y=0.0057$ at $Z=0.78$, the optimal value is $k_{\text {opt }} \approx 6$. In Fig. 4, we show the difference between the exact solution and the result of perturbation at $Z=0.84$ and $Z=0.78$ as a function of the order in perturbation theory in SU(3). The origin of the divergence are non-perturbative terms that do not contain in a Taylor expansion $[43,44]$. 
Fig. 3 Left panel: The ratio of the four-point function as a function of $Z$ for $S U(3)$. Right panel: The $Z$ dependence of $y=\bar{\mu}^{4} / \kappa a^{2}$ for different orders in perturbation theory. The full circles represent the result of the non-perturbative calculation
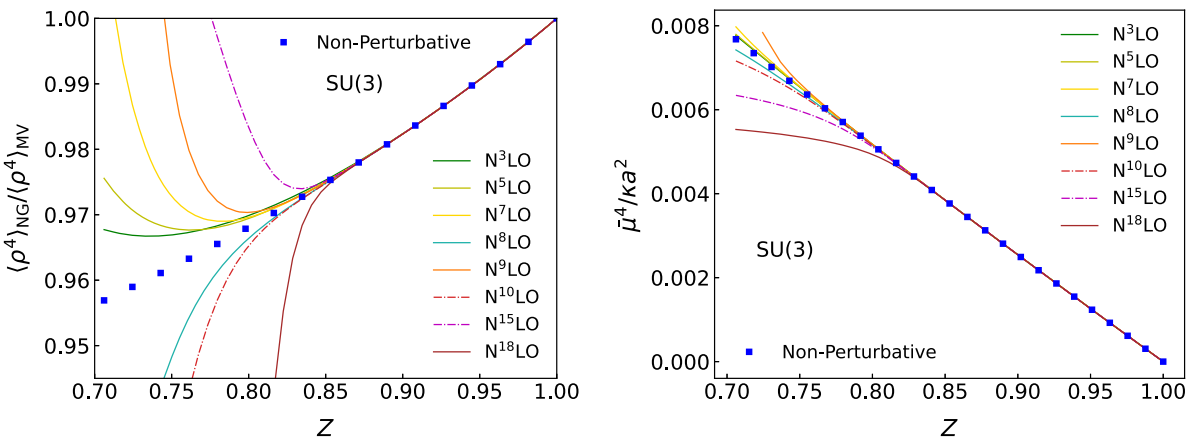

Fig. 4 The difference between the exact solution and the $\mathrm{N}^{n} \mathrm{LO}$ calculations for $\mathrm{SU}(3)$ at $Z=0.84$ (left panel) and $Z=0.78$ (right panel)

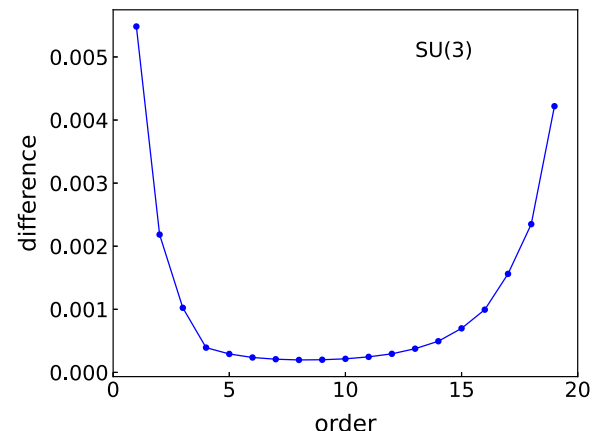

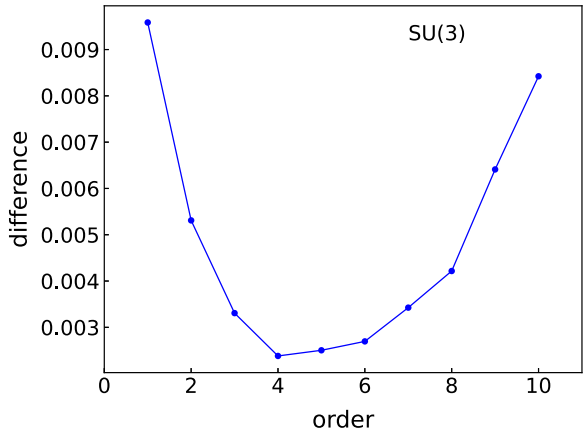

4 Perturbation theory in the limit of large non-Gaussian fluctuations: the $\mathrm{N}^{n} \mathrm{LO}$ order

In this section, we consider an arbitrary order in perturbation theory in the limit of large non-Gaussian fluctuations. The integral for the evaluation of each order in this regime is given by

$N_{n} \equiv \frac{1}{\mathcal{N}_{0}} \int \prod_{a} d \rho_{x}^{a} \rho_{x}^{n} e^{-S_{W}}=\frac{\int d r r^{N_{c}^{2}-2+n} e^{-S_{r}}}{\int d r r^{N_{c}^{2}-2} e^{-S_{r}}}$,

where $S_{W}=\int d^{2} \rho_{x} \rho_{x}^{4} / \kappa$ and $S_{r}=a^{2} r^{4} / \kappa$. By using

$$
\int_{0}^{\infty} d x x^{n} \exp \left[-\frac{x^{4}}{\kappa}\right]=\frac{1}{4} \Gamma\left(\frac{n+1}{4}\right) \kappa^{\frac{n+1}{4}}
$$

and $\Gamma(z+1)=z \Gamma(z)$, the integral in Eq. (48) yields:

$$
\begin{aligned}
N_{n} & =\frac{\left(N_{c}^{2}-1\right)}{4} \frac{\Gamma\left(\left(N_{c}^{2}-1+n\right) / 4\right)}{\Gamma\left(\left(N_{c}^{2}+3\right) / 4\right)}\left(\frac{\kappa}{a^{2}}\right)^{n / 4} \\
& =\frac{\left(N_{c}^{2}+n-5\right)}{4} \frac{\kappa}{a^{2}} N_{n-4},
\end{aligned}
$$

with the initial conditions $N_{0}=1$ and

$$
\begin{aligned}
N_{2} & =\frac{\Gamma\left(\left(N_{c}^{2}+1\right) / 4\right)\left(\kappa / a^{2}\right)^{\left(N_{c}^{2}+1\right) / 4}}{\Gamma\left(\left(N_{c}^{2}-1\right) / 4\right)\left(\kappa / a^{2}\right)^{\left(N_{c}^{2}-1\right) / 4}} \\
& =\frac{\left(N_{c}^{2}-1\right)}{4} \frac{\Gamma\left(\left(N_{c}^{2}+1\right) / 4\right)}{\Gamma\left(\left(N_{c}^{2}+3\right) / 4\right)} \frac{\sqrt{\kappa}}{a} .
\end{aligned}
$$

By using Eq. (50) one can calculate the two- and -four point function of color charges for an arbitrary order $\mathcal{O}\left(Z^{n}\right)$ in perturbation theory:

$$
\begin{aligned}
\left\langle\rho_{x}^{a} \rho_{x}^{a}\right\rangle & =\left(\sum_{k=0}^{n} \frac{1}{k !}(-x)^{k} N_{4 k+2}\right)\left(\sum_{k=0}^{n} \frac{1}{k !}(-x)^{k} N_{4 k}\right)^{-1} \\
& =\left(N_{c}^{2}-1\right) \frac{\sqrt{\kappa}}{a} \sum_{k=0}^{n} d_{k} w^{k} \\
\left\langle\rho_{x}^{a} \rho_{x}^{a} \rho_{x}^{b} \rho_{x}^{b}\right\rangle & =\left(\sum_{k=0}^{n} \frac{1}{k !}(-x)^{k} N_{4 k+4}\right)\left(\sum_{k=0}^{n} \frac{1}{k !}(-x)^{k} N_{4 k}\right)^{-1} \\
& =\frac{\left(N_{c}^{2}-1\right)}{4} \frac{\kappa}{a^{2}}\left(1-2 \sum_{k=0}^{n-1} d_{k} w^{k+1}\right)
\end{aligned}
$$

where $x=Z a^{2} / 2 \bar{\mu}^{2}$ and $w=\sqrt{\kappa} x / a=Z a \sqrt{\kappa} / 2 \bar{\mu}^{2}$. The coefficients $d_{k}$ are listed in the Appendix D. The requirement of matching the two-point function of color charges of the MV model yields the following constraint

$2 \sum_{k=0}^{n} d_{k} w^{k+1}=Z$

at the order $\mathcal{O}\left(Z^{n}\right)$. Thus, by noting that $w^{2}=Z^{2} / 4 y$, the ratio of the four-point function of color charges in the nonGaussian and Gaussian theories is given by

$R_{n}=\frac{w^{2}}{\left(N_{c}^{2}+1\right) Z^{2}}\left(1-2 \sum_{k=0}^{n-1} d_{k} w^{k+1}\right)$ 


$$
\approx \frac{w^{2} / Z^{2}}{N_{c}^{2}+1}(1-Z)=\frac{1-Z}{4\left(N_{c}^{2}+1\right) y},
$$

which is the same as Eq. (45) that is obtained by the expansion in $1 / \kappa$. In the $Z \rightarrow 0$ limit, we have $y=d_{0}^{2}$ then,

$R_{0}=\frac{1}{4\left(N_{c}^{2}+1\right) d_{0}^{2}}=\frac{4 \Gamma\left(\left(N_{c}^{2}+3\right) / 4\right)^{2}}{\left(N_{c}^{2}+1\right) \Gamma\left(\left(N_{c}^{2}+1\right) / 4\right)^{2}}$,

which is consistent with our previous work [29]. Lastly, at the order $\mathcal{O}(Z)(\mathrm{LO}), R_{1}$ is given by

$$
\begin{aligned}
R_{1} & =\frac{\left(-d_{0}+D\right)^{2}}{4\left(N_{c}^{2}+1\right) d_{1}^{2} Z^{2}}\left(1+\frac{d_{0}}{d_{1}}\left(d_{0}-D\right)\right), \\
D & =\sqrt{d_{0}^{2}+2 d_{1} Z},
\end{aligned}
$$

recovering the result presented in [29].

Let us now compare results from both perturbative calculations worked out in this and previous sections to the full non-perturbative (numerical) calculation. The left panel of Fig. 5 shows the ratio of the four-point function of color charges in the non-Gaussian and the Gaussian theories for $\mathrm{SU}(3)$ and $\mathrm{SU}(6)$ from the perturbation in $1 / \kappa \rightarrow 0$ (dashed lines) and $Z \rightarrow 0$ (solid lines), corresponding to small and large deviations from the MV model, respectively. The perturbation in $Z$ shows a good agreement with the nonperturbative calculation up to $Z=0.7$ for $\mathrm{SU}(3)$. We showed in [29] that deviations from the MV model for $\left\langle\rho_{x}^{a} \rho_{x}^{a} \rho_{x}^{b} \rho_{x}^{b}\right\rangle$ when $Z \rightarrow 0$ - which is the region where one finds maximum deviation in both theories for this particular correlator - become smaller as $N_{c}$ increases (with differences fully disappearing in the limit of large $N_{c}$ ). Thus, by increasing $N_{c}$ we expect that the perturbation in $1 / \kappa$ becomes better while the perturbation in $Z$ becomes worse. Indeed, comparing the results for $\mathrm{SU}(3)$ and $\mathrm{SU}(6)$ confirms this expectation. In the right panel of Fig. 5, we also compare $Z$ dependence of $y=\bar{\mu}^{4} / \kappa a^{2}$ with the non-perturbative results obtained by numerical integration. The range of agreement between the different perturbative calculations and non-perturbative one for the $Z$ dependence of $y$ is similar to the range of agreement for the four-point function of color charges.

\section{The Borel-Padé resummation}

The previous sections showed that the perturbative series for the four-point function of color charges in both the limit of small and large non-Gaussian fluctuations diverges. In this section, we employ the Borel-Padé resummation method [47] to construct a convergent series for our twoand four-point functions of color charges.

The procedure of the Borel-Padé resummation is the following. Let $Z(y)=\sum_{n=0}^{\infty} c_{n} y^{n}$ be a divergent series, where $c_{n}$ are the coefficients of the perturbative series and $y$ is the expansion parameter. As a first step, one calculates the Boreltransformed coefficients: $b_{n}=c_{n} / n$ ! to remove the factorial growth of each coefficient, which is a typical reason for the divergent series. The coefficients $b_{n}$ define the Boreltransformed series: $B(\tau)=\sum_{n=0} b_{n} \tau^{n}$. In the Borel-Padé summation, Padé approximants are then used to approximate the Borel sum by a rational function:

$B_{L / M}(\tau)=\frac{\sum_{n=0}^{L} p_{n} \tau^{n}}{1+\sum_{n=1}^{M} q_{n} \tau^{n}}$,

where $N=L+M$ gives the order of the Padé approximant. The coefficients $q_{n}$ and $p_{n}$ are found by equating order by order the Taylor series of $B(\tau)$. Then one performs the Laplace transformation,

$Z_{B, L / M}(y)=\int_{0}^{\infty} e^{-\tau} B_{L / M}(\tau y) d \tau$,

in order to reintroduce the contribution of the factorial factors removed in the first step.

As an example, let us consider the perturbative series in the limit of small non-Gaussian fluctuations for $L=M=1$, in which we solve Eq. (41) up to the order of $N=2$ :

$Z=1-4\left(N_{c}^{2}+1\right) y+32\left(N_{c}^{2}+1\right) y^{2}$.

The $[\mathrm{L} / \mathrm{M}]=[1 / 1]$ Padé approximant is given by

$B_{[1 / 1]}=\frac{1-4 N_{c}^{2} \tau}{1+4 \tau}$.

The value $y$ is obtained by solving

$Z=\int_{0}^{\infty} e^{-\tau} \frac{1-4 N_{c}^{2} \tau y}{1+4 \tau y} d \tau$

for a fixed value of $Z$ and the ratio of four-point functions of color charges is given by

$R_{[1 / 1]}=\int_{0}^{\infty} e^{-\tau} \frac{1+2\left(N_{c}^{2}+3\right) \tau y}{1+2\left(N_{c}^{2}+7\right) \tau y} d \tau$.

Higher-order approximants can be easily obtained in the Borel-Padé resummation method, usually providing a better approximation for the divergent series (in case the series is Borel-Padé summable).

Figure 6 shows a comparison of Borel-Padé approximants of different orders with the fully non-perturbative result for the ratio of the four-point function of color charges as a function of the renormalization factor, $Z$, for $\mathrm{SU}(3)$ (left panel) and SU(6) (right panel). As can be seen, the Borel-Padé resummation of order [2/2] can reproduce in a good approximation the entire $Z$ dependence of the ratio for $\mathrm{SU}(3)$ and $\mathrm{SU}(6)$, even though we only utilize information from the regime of small deviations from the MV model. A possible reason for this fast convergence is due to the small values of $y$, which are fixed by the condition Eq. (3) that the two-point functions of the non-Gaussian and the Gaussian theory are 
Fig. 5 Left panel: The ratio of the four-point function as a function of $Z$ for SU(3) and SU(6) group. Right panel: $Z$ dependence of $y=\bar{\mu}^{4} / \kappa a^{2}$
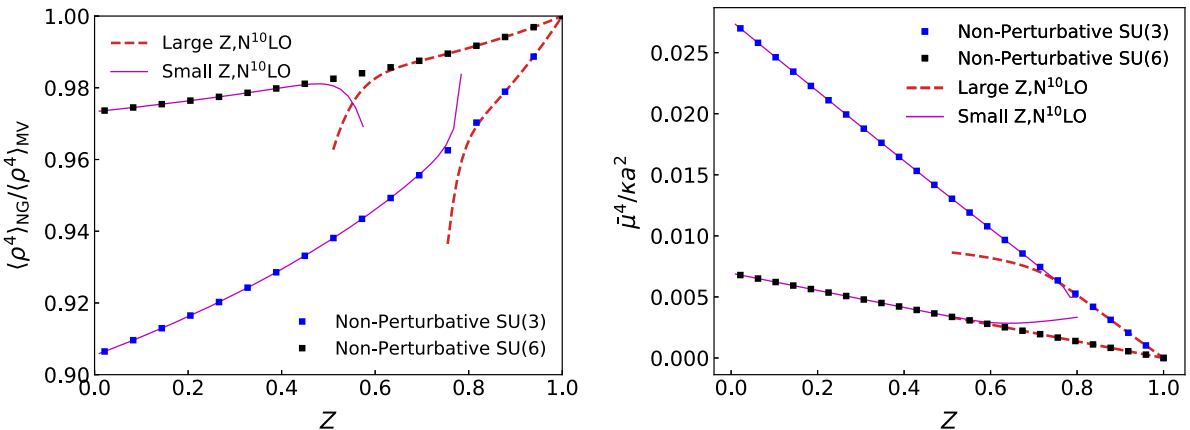

Fig. 6 The ratio of the four-point function as a function of $Z$ for $\mathrm{SU}(3)$ (left panel) and $\mathrm{SU}(6)$ group (right panel)

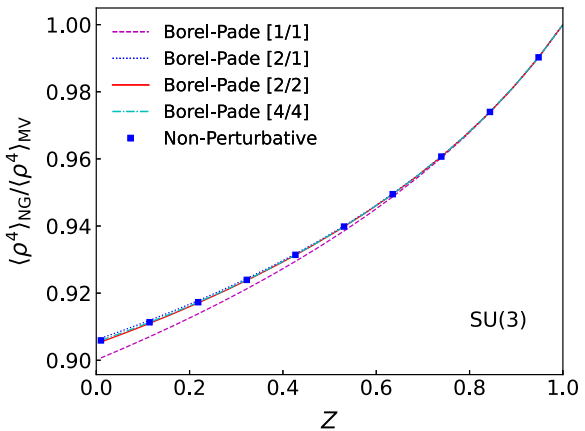

the same. The condition gives the value of $y=d_{0}^{2}=0.027$ at $Z=0$ for $\mathrm{SU}(3)$ as worked out in Sect. 4.

\section{Conclusions}

In this work, we considered the extension of the MV model by adding the first $\mathrm{C}$-even correction and studied the structure of the perturbative series for the two- and four-point function of color charges at $\mathrm{N}^{n} \mathrm{LO}$ orders in perturbation theory both in the limits of small and large non-Gaussian color charges fluctuations.

Starting with the regime of small deviations from the MV model, we first extended previous results for these correlators to NLO and NNLO in $1 / \kappa$ by explicitly calculating each Feynman diagram contributing at each one of these orders in perturbation. Next, a way to calculate these correlators at an arbitrary order, $\mathrm{N}^{n} \mathrm{LO}$, in $1 / \kappa$ was provided. We found a simple expression for the four-point function of color charges. Such a calculation allowed us to explicitly show that the resulting perturbative series is divergent; the optimal truncation for the perturbative series was estimated. A similar procedure to calculate the two- and four-point function of color charges at $\mathrm{N}^{n} \mathrm{LO}$ in the limit of large non-Gaussian color charges fluctuations has also been presented.

Lastly, we showed that the perturbative series in $1 / \kappa$ is Borel-Padé summable, and one is able to recover the nonperturbative result by employing a Borel-Padé approximant that only involves the first few terms of this series.
Extensions of the MV model of Gaussian color charges fluctuations may be relevant for describing small collision systems in the Color Glass Condensate framework. This model may potentially be relevant and useful for the modelling of energy-momentum correlations in the initial state of heavy-ion collisions [50], and of eccentricities and their fluctuations [51,52].

Acknowledgements We are grateful to Adrian Dumitru for his helpful comments, and careful reading of the manuscript. Y. N. acknowledges the support by the Grants-in-Aid for Scientific Research from JSPS (JP21K03577). A. V. G. acknowledges the support from FAPESP through the Grants 17/05685-2, 17/14974-8, 18/24720-6 and 21/04924-9.

Data Availability Statement This manuscript has associated data in a data repository. [Authors' comment: The calculations carried out in the manuscript have been explained in detail, which suffices to reproduce the results with additional data provided by Appendix C and D].

Open Access This article is licensed under a Creative Commons Attribution 4.0 International License, which permits use, sharing, adaptation, distribution and reproduction in any medium or format, as long as you give appropriate credit to the original author(s) and the source, provide a link to the Creative Commons licence, and indicate if changes were made. The images or other third party material in this article are included in the article's Creative Commons licence, unless indicated otherwise in a credit line to the material. If material is not included in the article's Creative Commons licence and your intended use is not permitted by statutory regulation or exceeds the permitted use, you will need to obtain permission directly from the copyright holder. To view a copy of this licence, visit http://creativecomm ons.org/licenses/by/4.0/.

Funded by SCOAP $^{3}$. 
Appendix A: The two- and four-point function of color charges at NNLO in the limit of small non-Gaussian fluctuations

The diagrams contributing for the two-point function at the next-to-next-to-leading order (NNLO) in the limit of small non-Gaussian fluctuations are:

(a) $y$

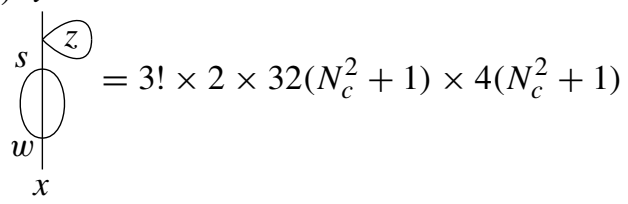

(b) $y$

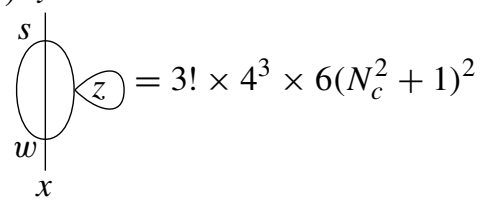

(c) $y$

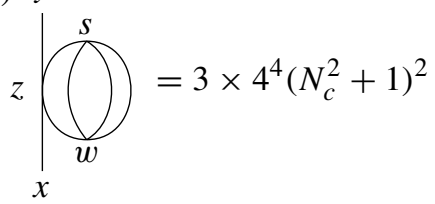

(d) $y$

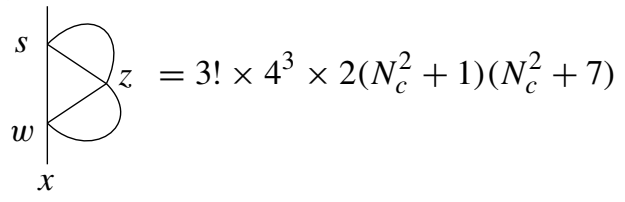

$y(\mathrm{e})$

$\begin{aligned} & w \\ & s \\ & z\end{aligned}=3 ! \times\left[4\left(N_{c}^{2}+1\right)\right]^{3}$

$x$

$y(\mathrm{f})$

$s\left(\mathbb{w}=3 ! \times\left[4\left(N_{c}^{2}+1\right)\right]^{3}\right.$

$x$

$y(\mathrm{~g})$

$s=3 ! \times 2 \times\left[4\left(N_{c}^{2}+1\right)\right]^{3}$

2

$x$

$$
\overbrace{x}^{y}=3 \times 2 \times 4^{3}\left(N_{c}^{2}+1\right)^{3}
$$

There are $3 !=6$ permutations of the three vertices $z-w-s$ except the diagrams (c) and (h). (c) and (h) have 3 distinguishable permutations for the three vertices $z-w-s$. The sum of the color factors yield:

$$
\begin{aligned}
\frac{\operatorname{sum}}{3 !}= & 64\left(N_{c}^{2}+1\right)\left[\left(5\left(N_{c}^{2}+1\right)^{2}\right.\right. \\
& \left.\left.+12\left(N_{c}^{2}+1\right)+2\left(N_{c}^{2}+7\right)\right)\right] \\
= & 64\left(N_{c}^{2}+1\right)\left(5 N_{c}^{4}+24 N_{c}^{2}+31\right),
\end{aligned}
$$

and the two-point function of color charges at NNLO order is given by:

$$
\begin{aligned}
\left\langle\rho_{x}^{a} \rho_{y}^{b}\right\rangle= & \mu^{2} \frac{\delta^{a b} \delta_{x y}}{a^{2}}\left[1-4 \frac{\mu^{4}}{\kappa a^{2}}\left(N_{c}^{2}+1\right)\right. \\
& +32 \frac{\mu^{8}}{\kappa^{2} a^{4}}\left(N_{c}^{2}+1\right)\left(N_{c}^{2}+2\right) \\
& \left.-64 \frac{\mu^{12}}{\kappa^{3} a^{6}}\left(N_{c}^{2}+1\right)\left(5 N_{c}^{4}+24 N_{c}^{2}+31\right)\right] .
\end{aligned}
$$

Thus, renormalized $\mu$ is defined as

$$
\begin{aligned}
\bar{\mu}^{2}= & \mu^{2}\left[1-4 \frac{\mu^{4}}{\kappa a^{2}}\left(N_{c}^{2}+1\right)+32 \frac{\mu^{8}}{\kappa^{2} a^{4}}\left(N_{c}^{2}+1\right)\left(N_{c}^{2}+2\right)\right. \\
& \left.-64 \frac{\mu^{12}}{\kappa^{3} a^{6}}\left(N_{c}^{2}+1\right)\left(5 N_{c}^{4}+24 N_{c}^{2}+31\right)\right] .
\end{aligned}
$$

The connected diagrams for four-point function at order $1 /\left(3 ! \kappa^{3}\right)$ are:
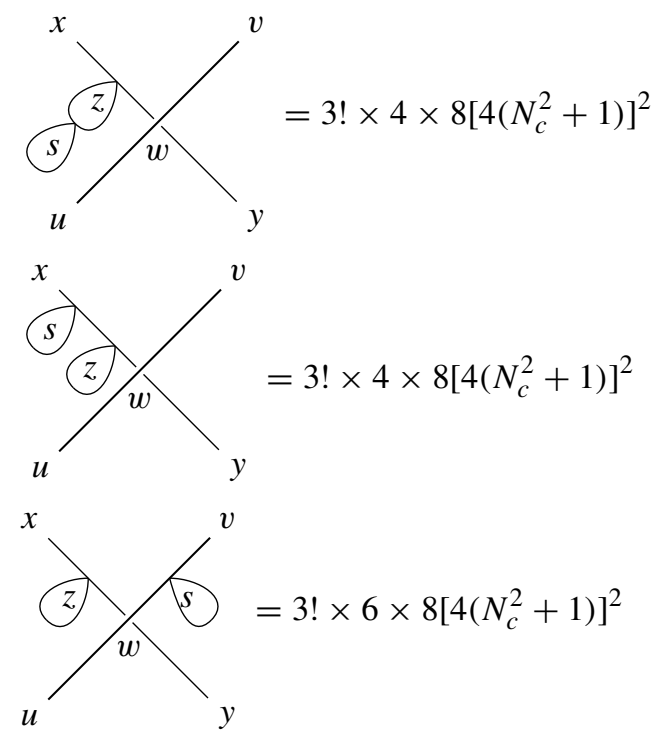

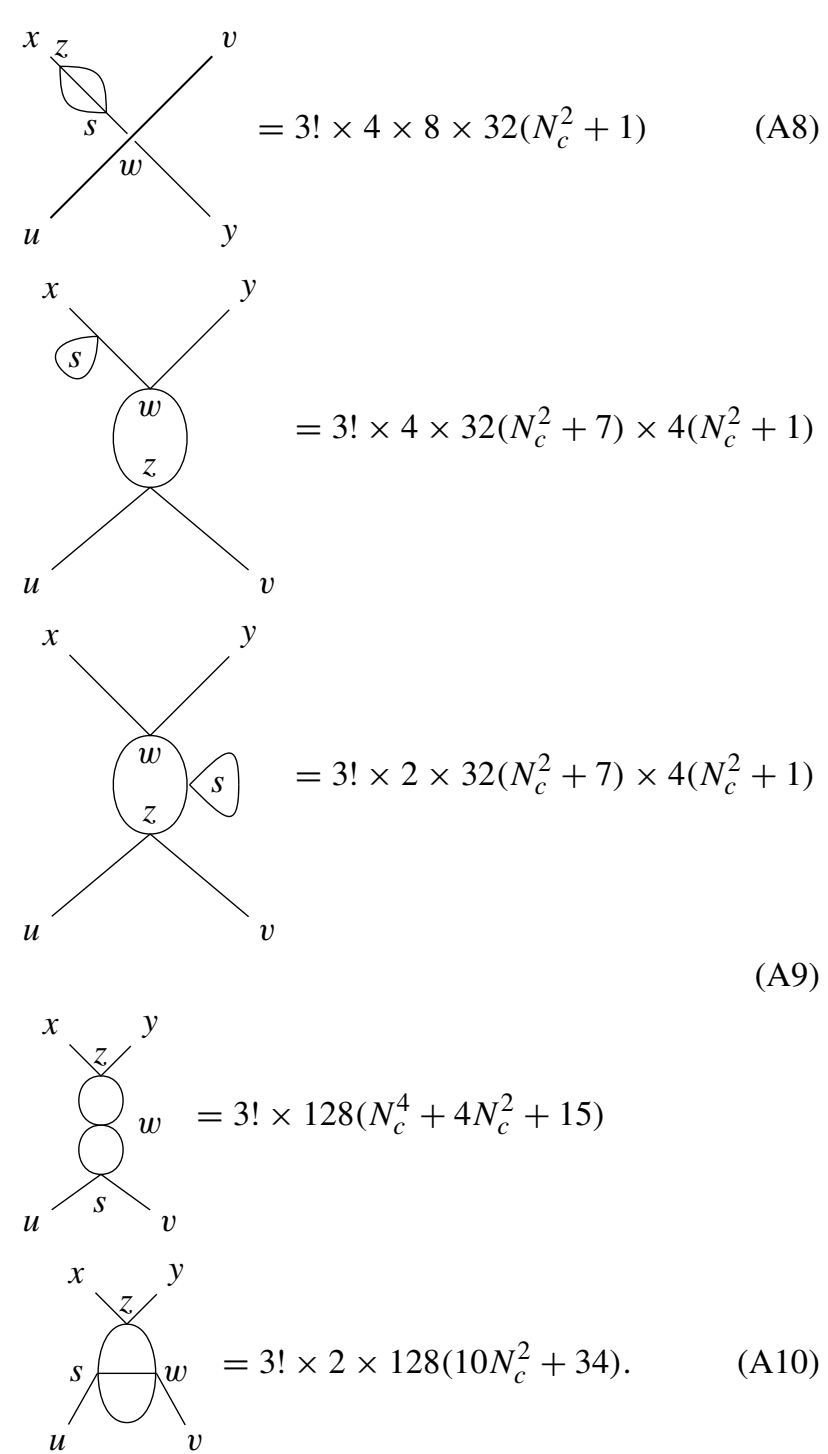

(A10)

We note that these connected graphs are also obtained by cutting the graphs of the two-point function.

$$
\begin{aligned}
\frac{\text { sum }}{3 ! \kappa^{3}}= & 128\left[16\left(N_{c}^{2}+1\right)^{2}+8\left(N_{c}^{2}+1\right)\right. \\
& +6\left(N_{c}^{2}+1\right)\left(N_{c}^{2}+7\right)+N_{c}^{4} \\
& \left.+4 N_{c}^{2}+15+2\left(10 N_{c}^{2}+34\right)\right] \\
= & 128\left(21 N_{c}^{4}+108 N_{c}^{2}+147\right) .
\end{aligned}
$$

$$
\begin{aligned}
\frac{\text { total sum }}{3 ! \kappa^{3}}= & 128\left(7 N_{c}^{6}+37 N_{c}^{4}\right. \\
& \left.+65 N_{c}^{2}+35+21 N_{c}^{4}+108 N_{c}^{2}+147\right) \\
= & 128\left(7 N_{c}^{6}+58 N_{c}^{4}+173 N_{c}^{2}+182\right) .
\end{aligned}
$$

The renormalized expression at $1 / \kappa^{3}$ is obtained as

$$
\begin{aligned}
A_{\text {con }}= & \frac{\mu^{4}}{a^{4}}\left[-8 \frac{\mu^{4}}{\kappa a^{2}}+32 \frac{\mu^{8}}{\kappa^{2} a^{4}}\left(5 N_{c}^{2}+11\right)\right. \\
& \left.-128 \frac{\mu^{12}}{\kappa^{3} a^{6}}\left(21 N_{c}^{4}+108 N_{c}^{2}+147\right)\right]
\end{aligned}
$$

$$
\begin{aligned}
= & \frac{\bar{\mu}^{4}}{a^{4}}\left[-8 \frac{\bar{\mu}^{4}}{\kappa a^{2}}+32 \frac{\bar{\mu}^{8}}{\kappa^{2} a^{4}}\left(N_{c}^{2}+7\right)\right. \\
& \left.-128 \frac{\bar{\mu}^{12}}{\kappa^{3} a^{6}}\left(N_{c}^{4}+24 N_{c}^{2}+83\right)\right] .
\end{aligned}
$$

\section{Appendix B: Gaussian integral for $\boldsymbol{n}$-th order}

The two- and four-point functions can be obtained by means of one-dimensional Gaussian integrations. We define $N_{n}$ as

$N_{n}=\frac{1}{\mathcal{N}_{0}} \int \prod_{a} d \rho_{x}^{a} \rho_{x}^{n} e^{-S_{G}}$.

Using the following Gaussian integral

$n_{n}=\int_{0}^{\infty} d x x^{n} \exp \left[-\frac{a^{2} x^{2}}{2 \mu^{2}}\right]=\frac{1}{2} \Gamma\left(\frac{n+1}{2}\right)\left(\frac{\sqrt{2} \mu}{a}\right)^{n+1}$.

The normalization is obtained as

$$
\begin{aligned}
n_{0} & =\frac{\sqrt{\pi}}{2}\left(\frac{\sqrt{2} \mu}{a}\right), \\
\mathcal{N}_{0} & =\int \prod_{a} d \rho_{x}^{a} e^{-\rho_{x}^{2} a^{2} /\left(2 \mu^{2}\right)}=n_{0}^{N_{c}^{2}-1} .
\end{aligned}
$$

Then, for even $n$,

$$
\begin{aligned}
N_{n} & =\frac{1}{\mathcal{N}_{0}} \int \prod_{a} d \rho_{x}^{a} \rho_{x}^{n} e^{-S_{G}} \\
& =\frac{\int d r r^{N_{c}^{2}-2+n} e^{-a^{2} r^{2} /\left(2 \mu^{2}\right)}}{\int d r r^{N_{c}^{2}-2} e^{-a^{2} r^{2} /\left(2 \mu^{2}\right)}} \\
& =\frac{\Gamma\left(\frac{\left(N_{c}^{2}-1+n\right)}{2}\right)\left(\frac{\sqrt{2} \mu}{a}\right)^{N_{c}^{2}-1+n}}{\Gamma\left(\frac{\left(N_{c}^{2}-1\right)}{2}\right)\left(\frac{\sqrt{2} \mu}{a}\right)^{N_{c}^{2}-1}} \\
& =\prod_{k=1}^{n / 2}\left(N_{c}^{2}-1+n-2 k\right)\left(\frac{\mu}{a}\right)^{n} \\
& =\left(N_{c}^{2}-3+n\right) N_{n-2} \frac{\mu^{2}}{a^{2}},
\end{aligned}
$$

where we have used $\Gamma(z+1)=z \Gamma(z)$ to get

$$
\begin{aligned}
& \Gamma\left(\frac{N_{c}^{2}-1+n}{2}\right) \\
& \quad=\frac{N_{c}^{2}-1+n-2}{2} \Gamma\left(\frac{N_{c}^{2}-1+n-2}{2}\right) .
\end{aligned}
$$

The first few terms are given by

$$
N_{2}=\left(N_{c}^{2}-1\right)\left(\frac{\mu}{a}\right)^{2} \text {, }
$$




$$
\begin{aligned}
& N_{4}=\left(N_{c}^{2}+1\right) N_{2}\left(\frac{\mu}{a}\right)^{2}, \\
& N_{6}=\left(N_{c}^{2}+3\right) N_{4}\left(\frac{\mu}{a}\right)^{2}, \\
& N_{8}=\left(N_{c}^{2}+5\right) N_{6}\left(\frac{\mu}{a}\right)^{2}, \\
& N_{10}=\left(N_{c}^{2}+7\right) N_{8}\left(\frac{\mu}{a}\right)^{2} .
\end{aligned}
$$

The four-point function in the NLO may be obtained as

$$
\begin{aligned}
\left\langle\rho_{x}^{4}\right\rangle & =\frac{\int d r r^{N_{c}^{2}-2} r^{4} e^{-a^{2} r^{2} /\left(2 \mu^{2}\right)}\left[1-\frac{a^{2}}{\kappa} r^{4}+\frac{a^{4}}{2 \kappa^{2}} r^{8}\right]}{\int d r r^{N_{c}^{2}-2} e^{-a^{2} r^{2} /\left(2 \mu^{2}\right)}\left[1-\frac{a^{2}}{\kappa} r^{4}+\frac{a^{4}}{2 \kappa^{2}} r^{8}\right]} \\
& =\left(N_{4}-\frac{a^{2}}{\kappa_{4}} N_{8}+\frac{a^{4}}{2 \kappa_{4}^{2}} N_{12}\right)\left(1-\frac{a^{2}}{\kappa_{4}} N_{4}+\frac{a^{4}}{2 \kappa_{4}^{2}} N_{8}\right)^{-1} \\
& =N_{4}-\frac{a^{2}}{\kappa_{4}}\left(N_{8}-N_{4}^{2}\right)+\frac{a^{4}}{2 \kappa_{4}^{2}}\left(N_{12}-3 N_{4} N_{8}+2 N_{4}^{3}\right) .
\end{aligned}
$$

The last term in the above equation is evaluated as

$$
\begin{aligned}
& \frac{1}{2}\left(N_{12}-3 N_{4} N_{8}+2 N_{4}^{3}\right) \\
& =\frac{\mu^{8}}{2 a^{8}} N_{4}\left[(9)(7)(5)(3)-3(5)(3)(1)(-1)+2\left(N_{c}^{4}-1\right)^{2}\right] \\
& =\left(N_{c}^{4}-1\right) \frac{\mu^{12}}{a^{12}} 16\left(5 N_{c}^{4}+24 N_{c}^{2}+31\right)
\end{aligned}
$$

where $(n) \equiv\left(N_{c}^{2}+n\right)$. Thus, we get the same result shown in Sect. 3.2 after using Eq. (B10) into Eq. (B9) and factoring out $\left(N_{c}^{4}-1\right) \mu^{4} / a^{4}$, which is common to all terms.

\section{Appendix C: Coefficients of the perturbative series for small non-Gaussian color charge fluctuations}

We list the coefficients $c, a_{k}$, and $c_{k}$ appearing in the perturbative series for the two- and four-point function of color charges in the limit of small non-Gaussian color charge fluctuations. The ten first coefficients of the series before applying the renormalization procedure are:

$$
\begin{aligned}
c= & 4\left(N_{c}^{2}+1\right), \\
a_{0}= & 1, \\
a_{1}= & -8\left(N_{c}^{2}+2\right), \\
a_{2}= & 16\left(5 N_{c}^{4}+24 N_{c}^{2}+31\right), \\
a_{3}= & -128\left(7 N_{c}^{6}+58 N_{c}^{4}+173 N_{c}^{2}+182\right), \\
a_{4}= & 512\left(21 N_{c}^{8}+260 N_{c}^{6}\right. \\
& \left.+1306 N_{c}^{4}+3100 N_{c}^{2}+2873\right), \\
a_{5}= & -4096\left(33 N_{c}^{10}+562 N_{c}^{8}+4146 N_{c}^{6}\right. \\
& \left.+16312 N_{c}^{4}+33621 N_{c}^{2}+28486\right), \\
a_{6}= & 4096\left(429 N_{c}^{12}+9520 N_{c}^{10}\right.
\end{aligned}
$$

$$
\begin{array}{rl} 
& +95377 N_{c}^{8}+544768 N_{c}^{6}+1841863 N_{c}^{4} \\
& \left.+3437392 N_{c}^{2}+2719291\right), \\
a_{7}= & -32768\left(715 N_{c}^{14}+19898 N_{c}^{12}+257207 N_{c}^{10}\right. \\
& +1977490 N_{c}^{8}+9630017 N_{c}^{6}+29269870 N_{c}^{4} \\
& \left.+50652541 N_{c}^{2}+37921862\right), \\
a_{8}= & 131072\left(2431 N_{c}^{16}+82452 N_{c}^{14}\right. \\
& +1326100 N_{c}^{12}+13062468 N_{c}^{10} \\
& +85096170 N_{c}^{8}+370462908 N_{c}^{6} \\
& +1038627956 N_{c}^{4}+1692065772 N_{c}^{2} \\
& +1210080143), \\
a_{9}= & -1048576\left(4199 N_{c}^{18}+169766 N_{c}^{16}\right. \\
& +3306284 N_{c}^{14}+40291196 N_{c}^{12}+334606770 N_{c}^{10} \\
& +1939973736 N_{c}^{8}+7757897212 N_{c}^{6} \\
& +20392359076 N_{c}^{4}+31601443135 N_{c}^{2} \\
& +21735270226), \\
a_{10} & 2097152\left(29393 N_{c}^{20}+1391720 N_{c}^{18}\right. \\
& +32136215 N_{c}^{16}+471973040 N_{c}^{14} \\
& +4829037610 N_{c}^{12}+35560069248 N_{c}^{10} \\
& +188741774926 N_{c}^{8}+705359761744 N_{c}^{6} \\
& +1757875371253 N_{c}^{4}+2610819668248 N_{c}^{2} \\
& +1735955801003) .
\end{array}
$$

The ten first coefficients for the renormalized perturbative series are listed next.

$$
\begin{aligned}
c_{0}= & 1 \\
c_{1}= & -8 \\
c_{2}= & 32\left(N_{c}^{2}+7\right) \\
c_{3}= & -128\left(N_{c}^{4}+24 N_{c}^{2}+83\right) \\
c_{4}= & 512\left(N_{c}^{6}+55 N_{c}^{4}+571 N_{c}^{2}+1357\right) \\
c_{5}= & -2048\left(N_{c}^{8}+104 N_{c}^{6}\right. \\
& \left.+2266 N_{c}^{4}+14976 N_{c}^{2}+27933\right) \\
c_{6}= & 8192\left(N_{c}^{10}+175 N_{c}^{8}+6770 N_{c}^{6}\right. \\
& \left.+88886 N_{c}^{4}+438621 N_{c}^{2}+688971\right) \\
c_{7}= & -32768\left(N_{c}^{12}+272 N_{c}^{10}+16885 N_{c}^{8}\right. \\
& +377600 N_{c}^{6}+3564163 N_{c}^{4} \\
& \left.+14301296 N_{c}^{2}+19746759\right) \\
c_{8}= & 131072\left(N_{c}^{14}+399 N_{c}^{12}+37093 N_{c}^{10}\right. \\
& +1288331 N_{c}^{8}+20104579 N_{c}^{6}+150092653 N_{c}^{4}, \\
& +515838295 N_{c}^{2}+644057785 \\
c_{9}= & -524288\left(N_{c}^{16}+560 N_{c}^{14}\right. \\
& +74116 N_{c}^{12}+3754624 N_{c}^{10} \\
& +88827950 N_{c}^{8}+1072010352 N_{c}^{6}
\end{aligned}
$$




$$
\begin{aligned}
& +6711810900 N_{c}^{4}+20446471008 N_{c}^{2} \\
& +23543136377) \\
c_{10}= & 2097152\left(N_{c}^{18}+759 N_{c}^{16}\right. \\
& +137556 N_{c}^{14}+9706268 N_{c}^{12} \\
& +328292526 N_{c}^{10}+5895609522 N_{c}^{8} \\
& +58633472228 N_{c}^{6}+320047345068 N_{c}^{4} \\
& \left.+885046782489 N_{c}^{2}+953277309583\right) .
\end{aligned}
$$

\section{Appendix D: Coefficients of the perturbative series for large non-Gaussian color charge fluctuations}

We list the coefficients $d_{k}$ appearing in the perturbative series for the two- and four-point function of color charges in the limit of large non-Gaussian fluctuations.

$$
\begin{aligned}
d_{0}= & \frac{1}{4} \frac{\Gamma\left(\left(N_{c}^{2}+1\right) / 4\right)}{\Gamma\left(\left(N_{c}^{2}+3\right) / 4\right)} \\
d_{1}= & d_{0}^{2}\left(N_{c}^{2}-1\right)-\frac{1}{4} \\
d_{2}= & d_{0}^{3}\left(N_{c}^{2}-1\right)^{2}-\frac{d_{0}}{4}\left(N_{c}^{2}-2\right) \\
d_{3}= & d_{0}^{4}\left(N_{c}^{2}-1\right)^{3}-\frac{d_{0}^{2}}{3}\left(N_{c}^{2}-2\right)\left(N_{c}^{2}-1\right) \\
+ & \frac{1}{48}\left(N_{c}^{2}-3\right), \\
d_{4}= & d_{0}^{5}\left(N_{c}^{2}-1\right)^{4}-\frac{5 d_{0}^{3}}{12}\left(N_{c}^{2}-2\right)\left(N_{c}^{2}-1\right)^{2} \\
& +\frac{d_{0}}{96}\left(2 N_{c}^{2}-5\right)\left(2 N_{c}^{2}-3\right), \\
d_{5}= & d_{0}^{6}\left(N_{c}^{2}-1\right)^{5}-\frac{d_{0}^{4}}{2}\left(N_{c}^{2}-2\right)\left(N_{c}^{2}-1\right)^{3} \\
& +\frac{d_{0}^{2}}{240}\left(N_{c}^{2}-1\right)\left(17 N_{c}^{4}-68 N_{c}^{2}+65\right) \\
& -\frac{1}{480}\left(N_{c}^{2}-3\right)\left(N_{c}^{2}-2\right) \\
d_{6}= & d_{0}^{7}\left(N_{c}^{2}-1\right)^{6}-\frac{7 d_{0}^{5}}{12}\left(N_{c}^{2}-2\right)\left(N_{c}^{2}-1\right)^{4} \\
& +\frac{7 d_{0}^{3}}{1440}\left(N_{c}^{2}-1\right)^{2}\left(22 N_{c}^{4}-88 N_{c}^{2}+85\right) \\
& -\frac{d_{0}}{5760}\left(N_{c}^{2}-2\right)\left(34 N_{c}^{4}-136 N_{c}^{2}+117\right) \\
& +\frac{1}{80640}\left(N_{c}^{2}-3\right)\left(17 N_{c}^{4}-68 N_{c}^{2}+63\right) \\
d_{7}= & d_{0}^{8}\left(N_{c}^{2}-1\right)^{7}-\frac{2 d_{0}^{6}}{3}\left(N_{c}^{2}-2\right)\left(N_{c}^{2}-1\right)^{5} \\
& \frac{d_{0}^{4}}{60}\left(N_{c}^{2}-1\right)^{3}\left(3 N_{c}^{2}-7\right)\left(3 N_{c}^{2}-5\right)
\end{aligned}
$$

$$
\begin{aligned}
& d_{8}= d_{0}^{9}\left(N_{c}^{2}-1\right)^{8}-\frac{3 d_{0}^{7}}{4}\left(N_{c}^{2}-2\right)\left(N_{c}^{2}-1\right)^{6} \\
&+\frac{d_{0}^{5}}{160}\left(N_{c}^{2}-1\right)^{4}\left(32 N_{c}^{4}-128 N_{c}^{2}+125\right) \\
&-\frac{11 d_{0}^{3}}{40320}\left(N_{c}^{2}-2\right)\left(N_{c}^{2}-1\right)^{2}\left(80 N_{c}^{4}-320 N_{c}^{2}+293\right) \\
&+\frac{d_{0}}{645120}\left(496 N_{c}^{8}-3968 N_{c}^{6}\right. \\
&\left.+11468 N_{c}^{4}-14128 N_{c}^{2}+6237\right), \\
& d_{9}= d_{0}^{10}\left(N_{c}^{2}-1\right)^{9}-\frac{5 d_{0}^{8}}{6}\left(N_{c}^{2}-2\right)\left(N_{c}^{2}-1\right)^{7} \\
&+\frac{d_{0}^{6}}{144}\left(N_{c}^{2}-1\right)^{5}\left(37 N_{c}^{4}-148 N_{c}^{2}+145\right) \\
&-\frac{d_{0}^{4}}{1512}\left(N_{c}^{2}-2\right)\left(N_{c}^{2}-1\right)^{3}\left(53 N_{c}^{4}-212 N_{c}^{2}+197\right) \\
&+\frac{d_{0}^{2}}{362880}\left(N_{c}^{2}-1\right)\left(691 N_{c}^{8}\right. \\
&\left.-5528 N_{c}^{6}+16106 N_{c}^{4}-20200 N_{c}^{2}+9180\right) \\
&-\frac{1}{1451520}\left(N_{c}^{2}-3\right)\left(N_{c}^{2}-2\right) \\
&\left(31 N_{c}^{4}-124 N_{c}^{2}+105\right), \\
& d_{10}= d_{0}^{11}\left(N_{c}^{2}-1\right)^{10}-\frac{11 d_{0}^{9}}{12}\left(N_{c}^{2}-2\right)\left(N_{c}^{2}-1\right)^{8} \\
&+\frac{11 d_{0}^{7}}{480}\left(N_{c}^{2}-1\right)^{6}\left(14 N_{c}^{4}-56 N_{c}^{2}+55\right) \\
&-\frac{11 d_{0}^{5}}{120960}\left(N_{c}^{2}-2\right)\left(N_{c}^{2}-1\right)^{4} \\
&\left(578 N_{c}^{4}-2312 N_{c}^{2}+2171\right) \\
&+\frac{11 d_{0}^{3}}{29030400}\left(N_{c}^{2}-1\right)^{2}\left(10256 N_{c}^{8}-82048 N_{c}^{6}\right. \\
&\left.+240256 N_{c}^{4}-304640 N_{c}^{2}+140895\right) \\
&-\frac{d_{0}}{116121600}\left(N_{c}^{2}-2\right)\left(\left(11056 N_{c}^{8}-88448 N_{c}^{6}\right.\right. \\
&\left.252064 N_{c}^{4}-300672 N_{c}^{2}+126945\right), \\
&(\mathrm{D} 10) \\
&
\end{aligned}
$$

\section{References}

1. E. Iancu, R. Venugopalan, in Quark Gluon Plasma, ed. by R.C. Hwa et al., pp. 249-3363. arXiv:hep-ph/0303204

2. F. Gelis, E. Iancu, J. Jalilian-Marian, R. Venugopalan, Annu. Rev. Nucl. Part. Sci. 60, 463 (2010)

3. L.D. McLerran, R. Venugopalan, Phys. Rev. D 49, 2233 (1994)

4. L.D. McLerran, R. Venugopalan, Phys. Rev. D 49, 3352 (1994)

5. L.D. McLerran, R. Venugopalan, Phys. Rev. D 50, 2225 (1994)

6. V. Khachatryan et al. (CMS Collaboration), J. High Energy Phys. 09, 091 (2010)

7. The ATLAS Collaboration, ATLAS-CONF-2015-027 (2015)

8. G. Aad et al. (ATLAS), Phys. Rev. Lett. 116(17), 172301 (2016)

9. V. Khachatryan et al. (CMS), Phys. Lett. B 765, 193-220 (2017)

10. S. Chatrchyan et al. (CMS), Phys. Lett. B 718, 795-814 (2013)

11. B. Abelev et al. (ALICE), Phys. Lett. B 719, 29-41 (2013) 
12. B.B. Abelev et al. (ALICE), Phys. Lett. B 726, 164-177 (2013)

13. G. Aad et al. (ATLAS), Phys. Rev. Lett. 110(18), 182302 (2013)

14. R. Aaij et al. (LHCb), Phys. Lett. B 762, 473-483 (2016)

15. A. Ohlson (ATLAS, ALICE, CMS and LHCb), Contribution to 3rd Large Hadron Collider Physics Conference (LHCP 2015) 31 Aug-5 Sept 2015. St. Petersburg, Russian Federation. arXiv:1901.00747 [nucl-ex]

16. A. Adare et al. (PHENIX), Phys. Rev. Lett. 111(21), 212301 (2013)

17. L. Adamczyk et al. (STAR), Phys. Lett. B 743, 333-339 (2015)

18. J. Adams et al. (STAR Collaboration), Phys. Rev. Lett. 95, 152301 (2005)

19. J. Putschke, J. Phys. G 34, S679 (2007)

20. A. Adare et al. (PHENIX Collaboration), Phys. Rev. C 78, 014901 (2008)

21. B.I. Abelev et al. (STAR Collaboration), Phys. Rev. C 80, 064912 (2009)

22. B. Alver et al. (PHOBOS Collaboration), Phys. Rev. Lett. 104, 062301 (2010)

23. K. Dusling, W. Li, B. Schenke, Int. J. Mod. Phys. E 25(01), 1630002 (2016)

24. J.L. Nagle, W.A. Zajc, Annu. Rev. Nucl. Part. Sci. 68, 211-235 (2018)

25. S. Schlichting, P. Tribedy, Adv. High Energy Phys. 2016, 8460349 (2016)

26. S. Jeon, R. Venugopalan, Phys. Rev. D 70, 105012 (2004)

27. S. Jeon, R. Venugopalan, Phys. Rev. D 71, 125003 (2005)

28. A. Dumitru, J. Jalilian-Marian, E. Petreska, Phys. Rev. D 84, 014018 (2011)

29. A.V. Giannini, Y. Nara, Nucl. Phys. A 1010, 122178 (2021)

30. J. Jalilian-Marian, A. Kovner, L.D. McLerran, H. Weigert, Phys. Rev. D 55, 5414 (1997)

31. J. Jalilian-Marian, A. Kovner, A. Leonidov, H. Weigert, Nucl. Phys. B 504, 415 (1997)

32. J. Jalilian-Marian, A. Kovner, A. Leonidov, H. Weigert, Phys. Rev. D 59, 014014 (1998)

33. J. Jalilian-Marian, A. Kovner, H. Weigert, Phys. Rev. D 59, 014015 (1998)

34. J. Jalilian-Marian, A. Kovner, A. Leonidov, H. Weigert, Phys. Rev. D 59, 034007 (1999) [Erratum: Phys. Rev. D 59, 099903 (1999)]

35. A. Kovner, J.G. Milhano, Phys. Rev. D 61, 014012 (2000)
36. A. Kovner, J.G. Milhano, H. Weigert, Phys. Rev. D 62, 114005 (2000)

37. E. Iancu, A. Leonidov, L.D. McLerran, Nucl. Phys. A 692, 583 (2001)

38. E. Iancu, A. Leonidov, L.D. McLerran, Phys. Lett. B 510, 133 (2001)

39. E. Ferreiro, E. Iancu, A. Leonidov, L. McLerran, Nucl. Phys. A 703, 489 (2002)

40. A. Dumitru, J. Jalilian-Marian, Phys. Rev. D 81, 094015 (2010)

41. F.J. Dyson, Phys. Rev. 85, 631-632 (1952)

42. M. Mariño, Fortschr. Phys. 62, 455-540 (2014)

43. D.I. Kazakov, D.V. Shirkov, Fortschr. Phys. 28, 465-499 (1980)

44. M. Flory, R.C. Helling, C. Sluka, arXiv:1201.2714 [math-ph]

45. J. Zinn-Justin, Quantum Field Theory and Critical Phenomena, 3rd edn. (Clarendon Press, Oxford, 1996) (chapter 37)

46. C. Itzykson, J.B. Zuber, Quantum Field Theory (McGraw-Hill, New York, 1980) (section 9-4-1)

47. E. Caliceti, M. Meyer-Hermann, P. Ribeca, A. Surzhykov, U.D. Jentschura, Phys. Rep. 446, 1 (2007)

48. H. Mera, T.G. Pedersen, B.K. Nikolić, Phys. Rev. D 97(10), 105027 (2018)

49. H. Kleinert, A. Pelster, B. Kastening, M. Bachmann, Phys. Rev. E 62, 1537 (2000)

50. J.L. Albacete, P. Guerrero-Rodríguez, C. Marquet, JHEP 01, 073 (2019)

51. A. Dumitru, Y. Nara, Phys. Rev. C 85, 034907 (2012)

52. G. Giacalone, P. Guerrero-Rodríguez, M. Luzum, C. Marquet, J.Y. Ollitrault, Phys. Rev. C 100(2), 024905 (2019) 